\title{
Tuning of Particle Size in a Helical Coil Reactor
}

\author{
S. K. Pal, P. Dhasmana, K. D. P. Nigam, and V.Singh* \\ Department of Chemical Engineering, IIT Delhi, Hauz Khas, Delhi-110016, India \\ E-mail: vs225@chemical.iitd.ac.in
}

\section{Supplementary Information}

\section{XRD analysis for phase identification}

The XRD peaks of precipitated $\mathrm{BaSO}_{4}$ powder are matched with the standard inorganic reference database to check the purity of the precipitate. The diffraction pattern matched with the entry number 00-101-0542? ${ }^{?}$ of the COD (crystallographic open database) reference database which suggests an orthorhombic crystal system with cell dimensions of $a=8.8500$ $\AA, b=5.4300 \AA$ and $c=7.1300 \AA$. Fig. SS.1 shows the XRD pattern for the precipitated particle samples obtained at different Dean numbers.

\section{Zeta potential of barium sulfate in different solution conditions}

Table S.1: Zeta potential values obtained from the zetasizer ZS-90 for barium sulfate particles dispersed in different solutions.

\begin{tabular}{|l|c|c|}
\hline Sample No. & Dispersant & Zeta Potential $(\mathrm{mV})$ \\
\hline SW & DI water & -3.25 \\
SE & Pure Ethanol & -11.3 \\
SC1 & 0.1\% NaHMP in DI water & -12.8 \\
SC2 & $0.5 \%$ NaHMP in DI water & -68.2 \\
\hline
\end{tabular}




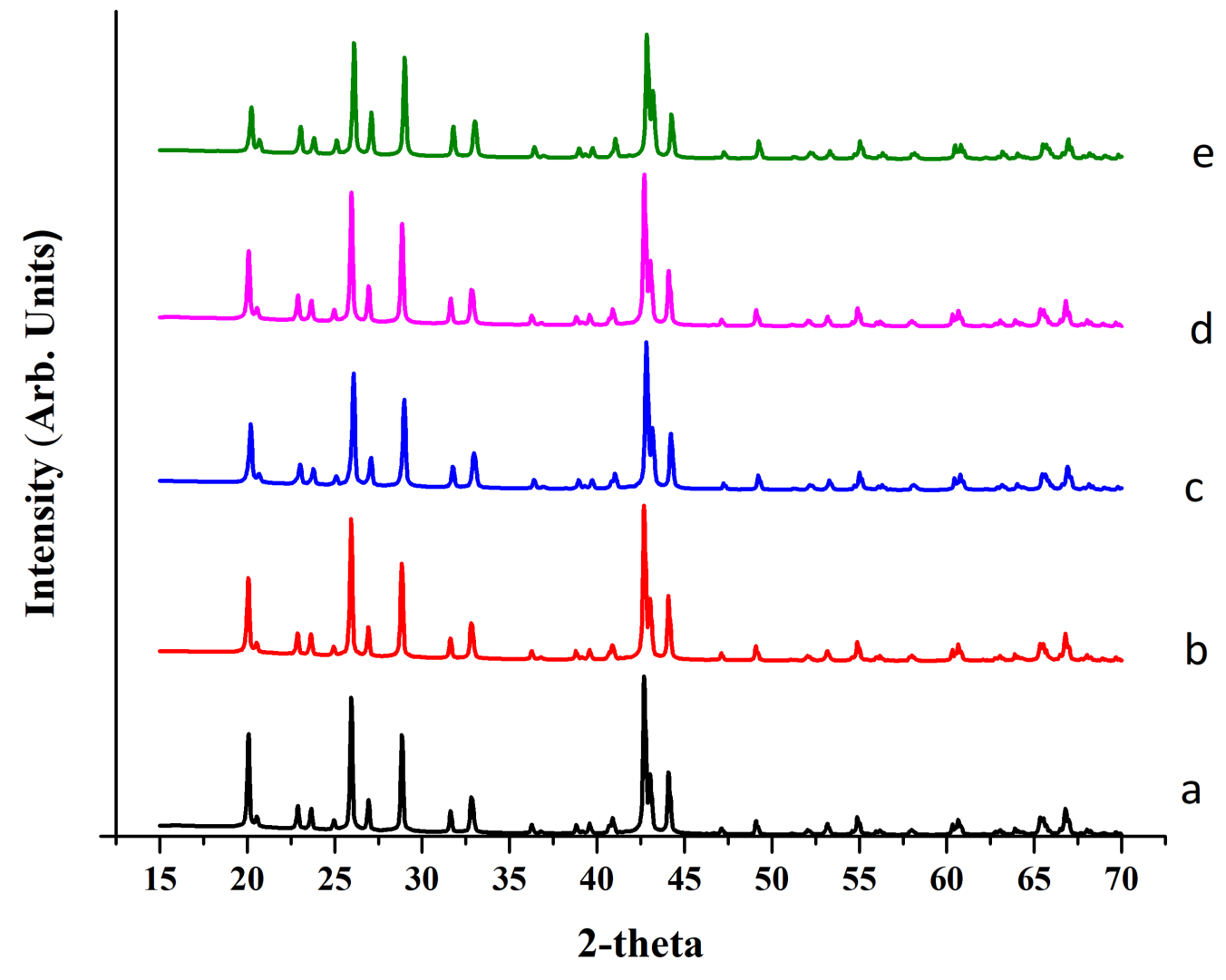

Figure SS.1: XRD patten of samples at different Dean numbers, (a) De=63.92, (b) De=133.66, (c) De=191.77, (d) De=290.56 and (e) De=370.5 respectively. 
Effect of concentration on polydispersity index and size in straight tube reactor

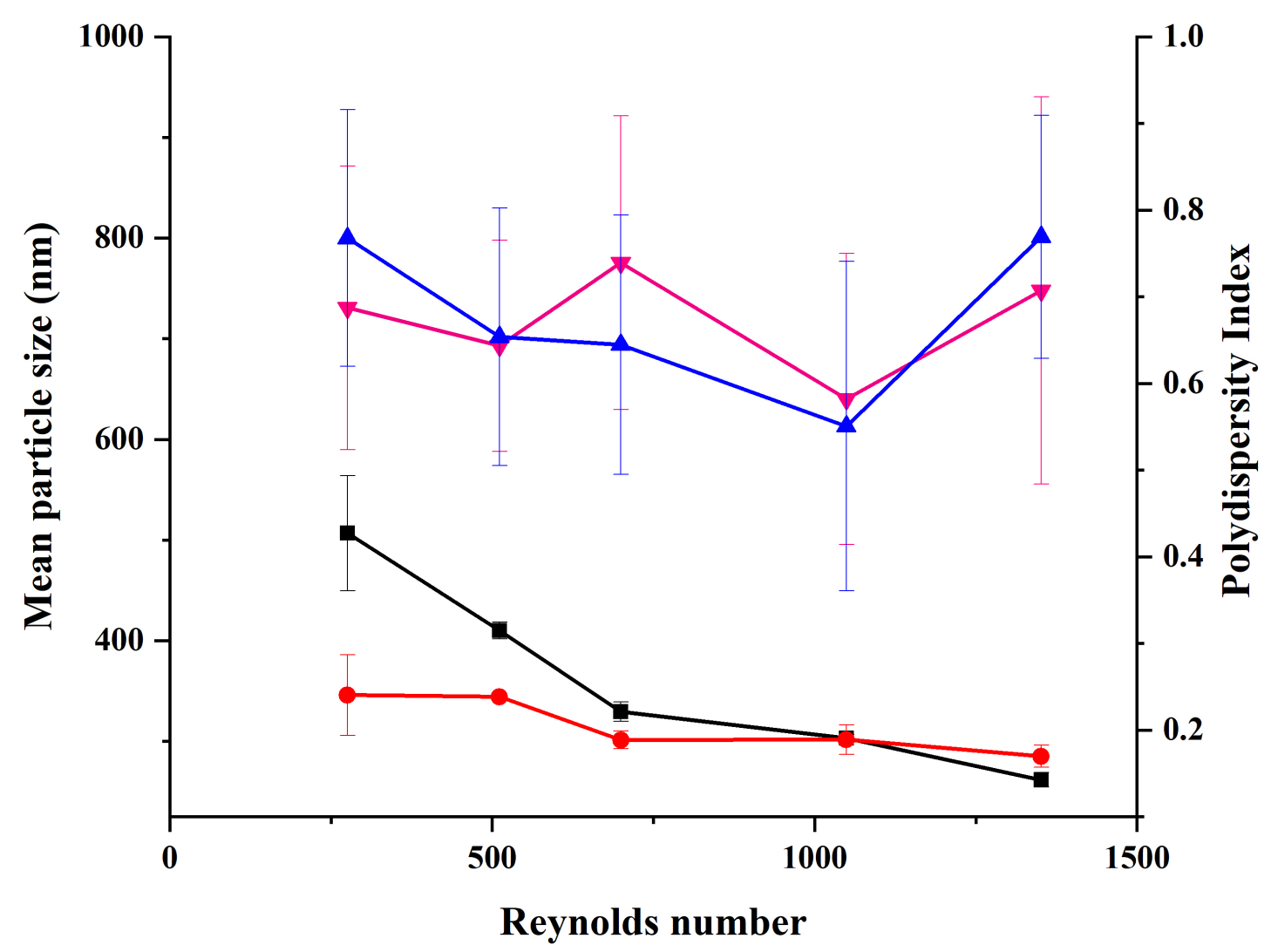

Figure SS.2: Effect of concentration on particle size and polydispersity index in a straight tube reactor where symbols represent $(\nabla)$ mean particle size at $0.1 \mathrm{M},(\mathbf{-})$ mean particle size at $0.5 \mathrm{M},(\mathbf{\Delta})$ polydispersity index at $0.1 \mathrm{M}$ and $(\bullet)$ Polydispersity index at $0.5 \mathrm{M}$ respectively. The difference in the particle size between a straight channel and a helical coil with the same Y-mixer further suggests that even at large concentrations the growth process is long compared to the time spent in the Y-mixer.

FESEM of particles with different flowrates and initial concentrations 


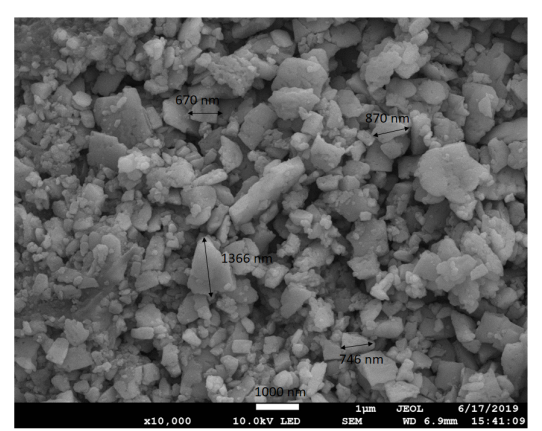

(a) FESEM image at $\mathrm{De}=63.93$ and $\mathrm{C}=0.1 \mathrm{M}$

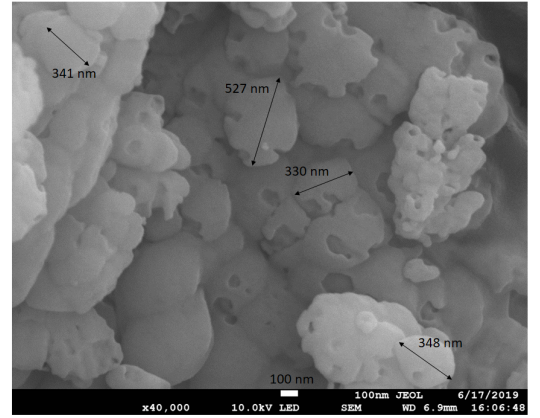

(b) FESEM image at $\mathrm{De}=370.5$ and $\mathrm{C}=0.1 \mathrm{M}$

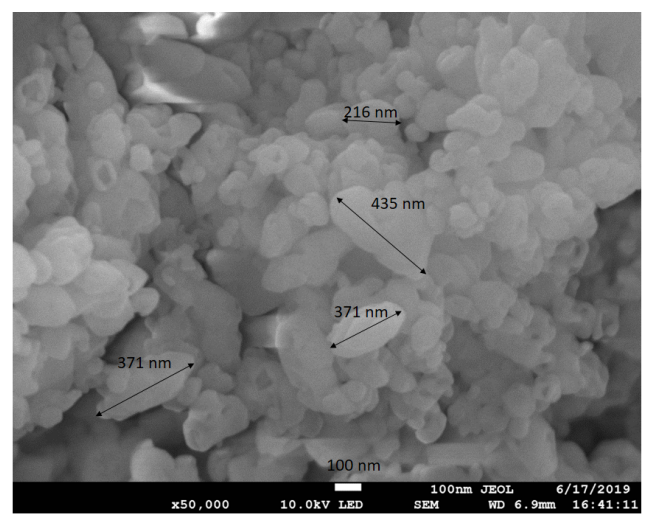

(c) FESEM image at $\mathrm{De}=370.5$ and $\mathrm{C}=0.5 \mathrm{M}$

Figure SS.3: FESEM images comparing particle size at different flowrates and initial reactant concentration conditions. 